\title{
Envisioning the Veracity of Digital Ecosystem in Improvising Effective Pandemic Response
}

\author{
Aditya Bharti ${ }^{1}$, Supriya Krishnan ${ }^{1,2 *}$ and Sudhanshu Kumar Bharti ${ }^{1,3 *}$ \\ ${ }^{1}$ Synergy Diabetes Research Foundation, Patna, India, ${ }^{2}$ Department of Personnel Management and Industrial Relations, \\ Darbhanga House, Patna University, Patna, India, ${ }^{3}$ Department of Biochemistry, Patna University, Patna, India
}

OPEN ACCESS

Edited by:

Natalie Elise Marler

Science Distributed, United States

Reviewed by:

Jason Goldwater

Atlas Research, United States

Iztok Perus,

University of Maribor, Slovenia

${ }^{*}$ Correspondence:

Supriya Krishnan

supriyadnr@rediffmail.com

Sudhanshu Kumar Bharti

sudhanshu_bharti@rediffmail.com

Specialty section

This article was submitted to Blockchain for Science, a section of the journal

Frontiers in Blockchain

Received: 27 August 2020 Accepted: 13 November 2020 Published: 10 February 2021

Citation:

Bharti A, Krishnan S and Bharti SK (2021) Envisioning the Veracity of

Digital Ecosystem in Improvising

Effective Pandemic Response.

Front. Blockchain 3:599428.

doi: 10.3389/fbloc.2020.599428
The obfuscation and the kind of cover-up or delay in COVID-19 crisis response put the veracity of global healthcare settings at stake and appended a biological dimension to geopolitical tensions. The ineffectual surveillance systems of public health and social measures cause the swift viral transmission pace amid mounting death toll and necessitate for an effective, cohesive, and strategic response. The digital ecosystem can serve the purposes intended in a transparent and immutable manner. This article highlights the problems encountered by the global healthcare settings in responding to pandemic and throws light on how the global digital ecosystem can handle crisis by managing the landscape radically through transparent information sharing via Internet of things (IOT) with the data being utilized by artificial intelligence (Al) and blockchain technologies on a cross-disciplinary collaborative basis. It will help to develop and provide borderless solutions of public health via monitoring, surveillance, detection, and prevention as well as digi-tool-assisted repurposed treatment by the use of authentic and decentralized distributed database that makes all contributors (participating countries, United Nations Organizations, the world medical associations, and global media and publications) accountable, inviolable, and efficient to tackle healthcare processes. It will extricate a blanket ban on information sharing thereby bringing democracy and freedom.

Keywords: blockchain, artificial intelligence, Internet of things, COVID-19 crisis, PHSM (public health and social measures), nodes, global ledger, MSDP (monitoring, surveillance, detection, and prevention)

\section{INTRODUCTION}

Today, the world reels under the devastating impact of the novel virus causing severe acute respiratory syndrome-corona virus-2 (SARS-CoV-2) or (COVID-19) pandemic. None of us have faced a threat like this before as it is perishing humanity and derailing the economies around the globe. As of May 27, 2020, a total of 5,488,825 COVID-19 positive cases have been registered worldwide with 349,095 fatalities (World Health Organization, 2020c).

In reality, the fast and transparent information sharing to the world about the provenance, virulence, and protective measures about the novel virus could have checked the exponential sweeping of the COVID-19 pandemic. The world would have been spared much of its present agony, and so many lives would not have been needlessly lost. Nearly 2 months after the first COVID-19 case was reported, we woke up from the darkness, but by this time, the killer virus has inflicted its wrath in Asia and aggravated human-to-human transmission further to its new epicenter, Europe, via potential asymptomatic, oblivious travelers with lax social habits about the 
contagion. The originating country stands in the dock, charged with misdemeanor concerning the health and well-being of humankind as the leadership obfuscated the extent of contagion and bungled the crisis response. In addition, the global healthcare organizations and authorities would have been a savior from the looming and rising menace, but their veracity today is also at stake for the kind of a cover-up or delay, resorted in the early days of the epidemic. This would not have been doable in an open society. All these episodes cause an exponential rise in COVID19 cases within 2 months of the first infection in early December and appended a biological dimension to geopolitical tensions.

To wrestle with one of the world's grave biological threats, a coordinated and transparent approach is considered necessary to be exercised in the overall response to the risk of infectious emergence or re-emergence with containment zones permitting safe investigative and research activities vital to disease prevention and developing management tools. Since the treatment of the COVID-19 is not known yet, prevention of transmission has been proposed to be one of the effective approaches to combat its progression and control as well as the comorbidities associated with it. To lessen its spread, most of the afflicted countries have banked on conventional methods of robust public health and social measures (PHSM) with monitoring, surveillance, detection, and prevention (MSDP). These measures range from travel restrictions to stay-at-home orders and intense quarantine actions to comprehensive contact tracing (to identify and manage people, exposed to a disease to prevent onward transmission) (World Health Organization, 2020b). All these measures also call for facilitating the delivery of important supplies to people in need by fueling self-reliance in the workforce assigned to deal with the humanitarian crises. This put in place the MSDP program for preparedness and readiness, supporting all the pillars of response actions as needed (https:// www.who.int/). Then again, frontier researches in biotechnology enabled with genomics, stem cell science, effective vaccine and drug development, bionics, and diagnostic technologies are contributing actively to the global research efforts for optimum healthcare delivery and treatment to deliver global public goods (Udugama et al., 2020).

Yet, these dealings are found to be ineffectual in tackling the pandemic as the virus is spreading swiftly amid mounting death toll and transmission pace. This is directly linked to security risk with centralized ownership of medical records, as the data are prone to obfuscation or hacking via the dark web, darknet, etc., and even a single data breach in a network can be destructive as evidenced earlier (Mirea et al., 2019). This may also be due to lack of promotion of regional and global collaboration as well as the vulnerable healthcare ecosystem due to limited access to quality care, patient safety, prohibitive drug prices, and uncertain reimbursement models and to some extent tampering, fraud, or political control. It is also evident that despite regulations like the Health Insurance Portability and Accountability Act (HIPAA) and the Health Information Technology for Economic and Clinical Health (HITECH) of USA and diverse healthcare organization bylaws are unable to protect the civil liberties from data breach front (Gold and McLaughlin, 2016). To prevent these from happening in the future, the digital ecosystem can solve the identity and vulnerability issues by decentralizing the health records as well as research and development data.

A leap forward to protect the civil liberties and bring trust to such organizations, which deal with sensitive records have to adapt a decentralized platform to channelize their data through public blockchain (as explained in Figure 2). For existing and future crisis, this platform can be kept improvising at the right time and trained properly with the data set, cohesive and geographically comprehensive strategic response to boost the conventional public health approaches of PHSM and MSDP through a distributed and adaptive digital system with properties of self-organization, scalability, and sustainability inspired from natural ecosystems (Briscoe and Wilde, 2006). The digital ecosystem can respond and lead the healthcare system based on innovation, advanced diagnosis, and rule-based therapies as well as lifting the implementation of eHealth (Car et al., 2017). Also, individuals can control their own data as a future civil right. The digital ecosystem includes the Internet of things (IoT) with next-generation telecommunication networks (e.g., 5G), big-data analytics, use of deep learning by artificial intelligence (AI), and blockchain technology (Ting et al., 2020).

The digital ecosystem includes IOT, AI, and blockchain (Figure 2). These interlinked digi-tools can be employed immutably for promptly acquiring, verifying, reporting, managing, sharing, and analyzing the stockpiling medical data/information with efficiency and transparency. These data (e.g., pandemic variable data, genomic data of individuals, etc.) are simultaneously explored to circumvent the disease recurrence by spotting its evolution, understanding the potential transmission drifts and its co-circulation with other viruses. This alleviates the consequence to healthcare settings, bringing up-to-date diagnostic tests and treatment for a disease with publicly accessible sequence database [e.g., global initiative on sharing all influenza data (GISAID) and GenBank] (World Health Organization, 2020c).

Today, the world is revolving on data generated by the IoT. The IoT is a network of Internet-connected physical devices including wireless and sensor technologies allowing them to collect and exchange massive datasets offering a plethora of applications via analytics and deep learning systems of AI, in healthcare domain. Taking into account the probability of rapid exponential proliferation of the pandemic, the PHSM and MSDP can be equipped with the IoT, which offers a platform that permits real-time updated data collection with variables for detection of new cases and clusters. These data sets are then reported to public health authorities via email, online entries, or mobile phone app, via SMS text message, or over the phone. These quantitative data include the actual number of people infected worldwide, daily entries of new cases, country-wise infection distribution as well as the disease severity in terms of recovered, active cases, critical cases, deceased ones, etc. The data can be collected at individual level, primary care level, community level, hospital settings levels, as well as through robust event-based surveillance (EBS) mechanisms. The EBS gathers raw unstructured data via formal and informal sources such as online content, radio broadcasts, and print media (World Health Organization, 2020b). 
These data are then filtered, triaged, verified, evaluated, and communicated with relevant content to the IoT devices and then to construe facility-based real-time surveillance data with big-data analytics and AI, transparently and impeccably (Atzori et al., 2010). The AI is the development of intelligence in machines, which can think and work like humans (e.g., learning, planning, and problem solving). Big data analytics with AI can deliver precise diagnosis and treatment with minimum resource addressing all the threats involved as well as to envisage effects (Topol, 2019). These tools offer prospects for executing modeling studies of viral activity as well as provide guidelines to healthcare policymakers to augment groundwork for the outbreak, public health planning and control. Moreover, these tools can improve the learning and communiqué about PHSM and MSDP by getting "real-time" precise updates about the disease and government initiatives on various societal platforms like Twitter and Facebook (Ting et al., 2020).

These results are then improved by blockchain technology. A blockchain is an open and decentralized, distributed ledger, used to record mounting real-time data-set in the form of blocks. These peer-to-peer network blocks are linked by cryptography with a set of connections of dispersed workstations in diverse organizations. New records can only be added based on distributed consensus of all the parties maintaining the database. Blockchain records transactions efficiently in a verifiable and consistent manner with personalized algorithms opposing data modification retroactively, without altering all subsequent blocks (Heaven, 2019). Moreover, AI and deep learning can augment the precise, specific, and inexpensive alternative diagnostic and screening tests based on large datasets of disease-positive cases. The AI algorithms are then used to trace the travel history of the suspects to affected places so that patients at higher risk could have been isolated. Most importantly, AI can also assist to discover novel drugs as well as to screen existing drugs that can be repurposed for treatment of COVID-19 (Fleming, 2018).

Further, maintaining the core and critical clinical service amidst pandemic is challenging, as the healthcare facilities ceases to function with closure of clinics and postponement of medical appointments. In such situation, to ensure patient's standard clinical care "virtual clinics" may well be arranged for tele-medicine consultations alleviating the clinical load of physicians. The biochemical test reports and imaging data (e.g., chest X-ray and/or CT scan of the thorax) are uploaded from peripheral sites and interpreted remotely, thereby reducing physical crowding of patients into hospitals. These virtual eplatforms could potentially be used for edification and research. From a supply chain point of view, blockchain can be tokenized for inimitable assets such as a patient's identity and, for general asset, like the number of available ventilators, personal protective equipment (PPE) inventories, to instruct manufacturers to build novel approved devices and how to distribute them to the needed locations with safety. Analyzing these captured data, smart contracts can be coded to recognize conditions and trigger rapid responses based on certain rules (Kaur and Oza, 2020).

Finally, by using blockchain coupled with artificial intelligence and other multitude of digital tools, healthcare settings could provide timely delivery of medicines to the doorsteps of patients with precise self-testing and tracking systems for existing and other burgeoning contagious malady. Diverse updated, structured, and unstructured data sets generated are aggregated into unified, federated, and easily accessible for applying advanced real-time analytics and AI. For example, the Epidemic Intelligence from Open Sources (EIOS) initiative of WHO is a network of diverse public health intelligence (PHI) worldwide, which create a unified all-hazards, one health solution approach to public health risks and threats, and subsequent intervention its ultimate goal (World Health Organization, 2020a).

To facilitate the transaction flow in blockchain, the actors involved include nodes, chain code, and global ledger. The nodes are generally simplified into quadrants based on its area of functionality. Here the four quadrants of nodes are participants who make a distributed network including (a) the participating countries, (b) United Nations Organizations (UNO), (c) the world medical associations (WMA), and (d) media and publications. The chain code is the common logics such as rules and regulations to be agreed upon by nodes before validation and execution of a transaction to share the information on the global ledger. The global ledger is the decentralized and distributed database (Figure 1). To make the network secure, the nodes can only talk to each other on the relevant ports and do not accept access from other nodes. There are a few ways to do this: (a) create firewall rules using cloud security policies, (b) create firewall rules on server [e.g., with iptables, (c) use a VPN, and (d) make the nodes listen only on local host on server and connect them using SSH tunnels]. More than 51\% attacks can be mitigated by choosing a proven consensus algorithm with high hashing power and having a legal penalty system for delayed block submission.

The country's medical organization CMO acts as a node of the network that makes a transaction for sharing the information of participant nodes like healthcare setting reports, PHSM data, etc. (Figure 1). Other nodes of workers and media also verify the transaction, and if it is found legitimate, then they validate and commit on the global ledger. Thus, a consensus approach of CMO, workers, and media rooted on civil liberties and healthcare ethics helps ensure the accuracy of the data being provided. Since it is associated with the previous transaction in the chain and hence a blockchain, this record is transparent and immutable. In some cases like pandemic, medical reports routed via healthcare ethics could be overshadowed with the protection of civil liberties. If the patient does not agree to disclose their identity, then by abiding the compliance of healthcare ethics and keeping his/her personal identity private, he/she has the option to opt for Zero Knowledge Proof (ZKP) for securing their identity and personal details while revealing only health monitoring data for the goods of healthcare research and prevent pandemic situation. An accredited research laboratory including P4 laboratory (laboratory accredited to work on class 4/highly dangerous pathogens, e.g., COVID-19 with necessary security safety with highly skilled staff), e.g., the Wuhan P4 laboratory of China and the Jean-Merieux laboratory, Lyon, France, with maximum-security and bio-safety facility approval, can also act as a node. Countries cannot conceal the medical data since every unique case has to be committed to a global ledger. Violating the 


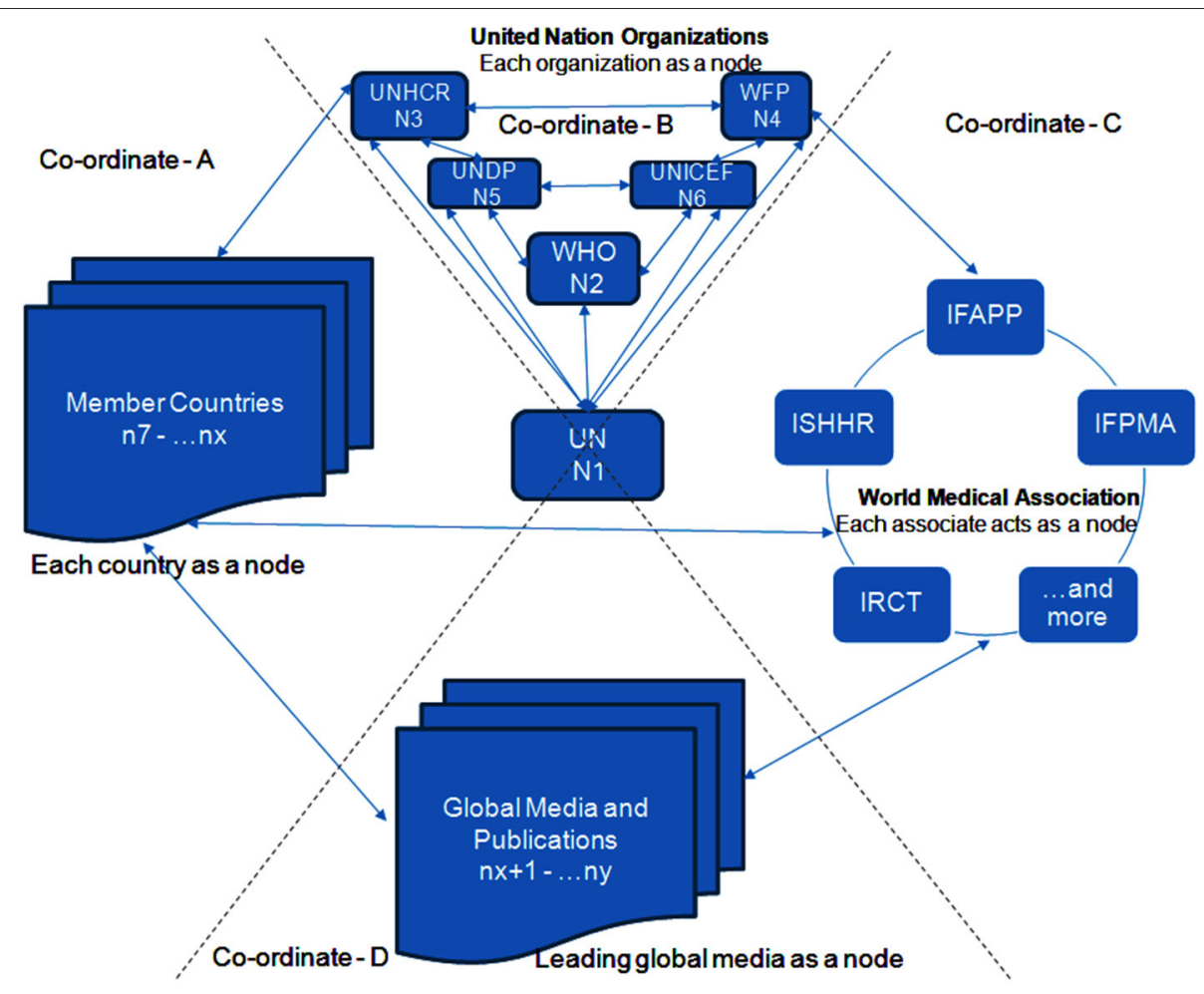

FIGURE 1 | The actors involved in blockchain are nodes (N) [a distributed network such as United Nations organizations (UNO), participant countries, global medical associations, media, and publications, which participate in sharing information], chain code (rules and regulations to be agreed upon by nodes before validation), and global ledger (execution platform of a transaction to share an immutable and updated information with the agreement of at least $51 \%$ of the nodes in the form of decentralized and distributed database to bring trust and transparency in the system). ABCD, simplified into quadrants based on node's area of functionality; UNHCR, The United Nations High Commissioner for Refugees; UNDP, The United Nations Development Programme; UNICEF, The United Nations Children's Fund; WHO, The World Health Organization; IFAPP, International Federation of Associations of Pharmaceutical Physicians; ISHHR, International Society for Health and Human Rights, IRCT, International Rehabilitation Council for Torture Victims; IHF, International Hospital Federation; IFPMA, International Federation of Pharmaceutical Manufacturers and Associations.

protocol, they will have to answer to the UN, WHO, as well as other countries.

The other nodes like world medical association (WMA) can provide the global progress with quality medical care and education rooted on high standards of professional competence and healthcare ethics (Beauchamp, 2013), and commit a transaction for the recommendations of drugs (World Medical Association, 2020) (Figure 2). If the nodes shared among peers are found legitimate, then they validate the transaction and update the global state of the ledger. The ledger grants the access of health monitoring data from IoT health monitoring service to the AI drug recommendation engine by mapping the patient ID as key. The AI drug recommendation engine conducts the research based on the history of chain data in the ledger health monitoring data from the IoT service. This chain data is additionally used by the AI-driven platform for tracing the history of the disease with future recommendations of drugs used in the past for its cure.

Further, nodes like United Nations Organization (UNO) and individual countries can commit transactions for the methods or steps to be taken to prevent the disease and release medical care and research fund related to the disease. The reconciled data are then vetted by the committees of the UNO and ensures that the new data matches the original data. The UNO also encourages validation by key healthcare leaders and experts who can report on discrepancies or questionable data. The UNOs like WHO and a country cannot have a private dealership after bringing this system into existence. Every data is transparent and immutable to the whole world. Irrespective of the country and their government, the global media and publications node insist on acting as an ombudsman, abiding by guiding principles of accountability and transparency. The media will act as a peer for secure communication platform with validity and improving social sharing dynamics and broadcasting, from day 1 to keep the whole world on the same updated page (Pazaitis et al., 2017).

Thus, the digital ecosystem offers (Shilo et al., 2020; Wikipedia., 2020; World Health Organization, 2020d,e) the automation, safety and accountability, to thwart corruption and facilitate well-organized services in handling public records. In addition, user-centric blockchain submissions permit end users to control, trace, and claim ownership of their shared content (Chakravorty and Rong, 2017). Thus, the seminal event of COVID-19 as a potential catalyst highlights the need of promotion of digital ecosystem for regional and 


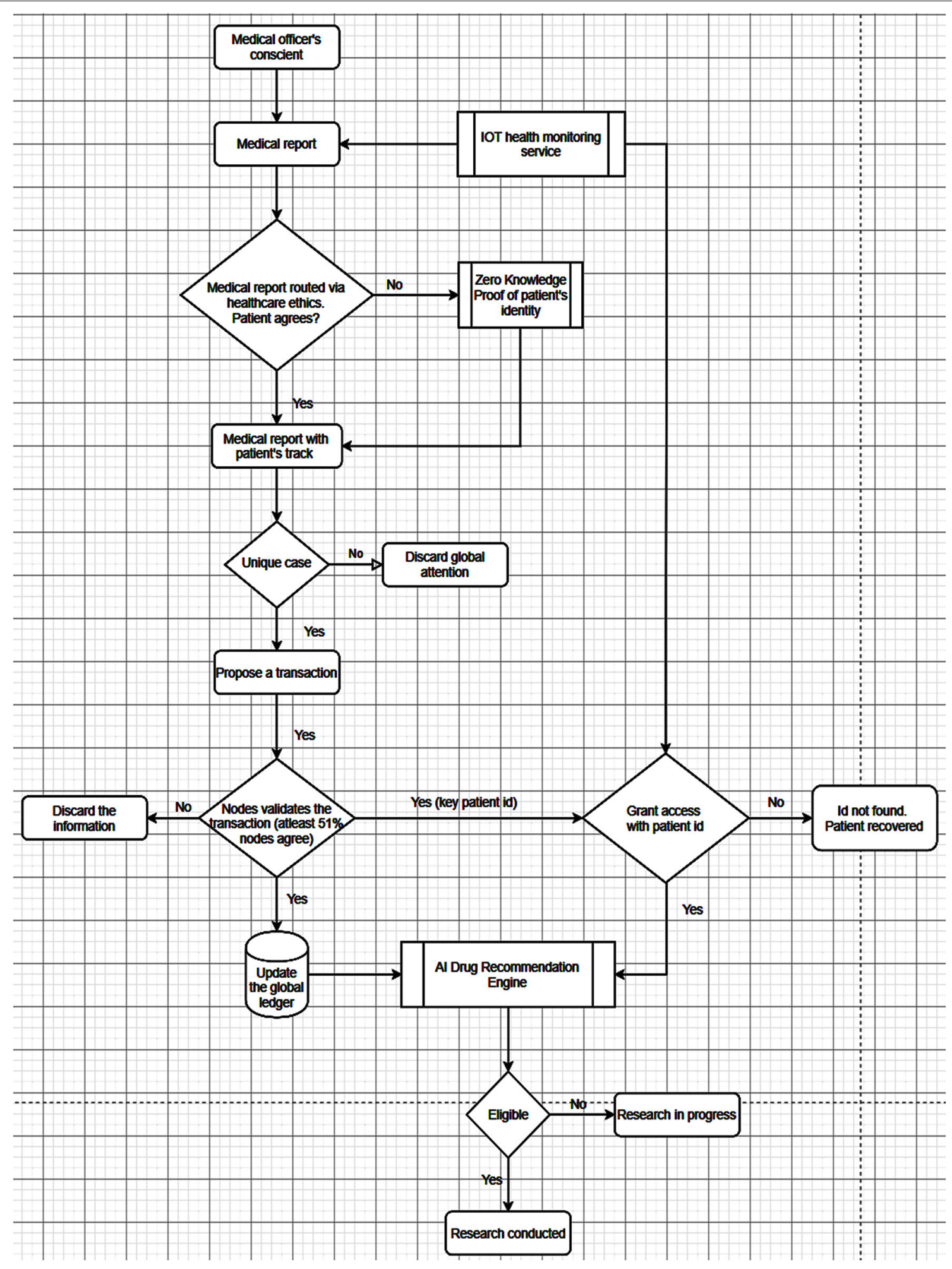

FIGURE 2 | A solution to overcome the misdemeanor at the setting/country/global health organization level and the authentication of news in global media/publication. A unique medical information/case is proposed as a transaction to the nodes to be shared among peers. If the nodes find it legitimate, then they validate the transaction and update the global state of the ledger. The ledger grants the access of health monitoring data to the Al Drug Recommendation Engine by mapping the patient's ID as key to delve into the history of chain data for future recommendations by tracing the drugs used in the past for its cure. Here A, B, C, and 
FIGURE 2 | D are transposable nodes of participant countries, UNO, World Medical Association, and Global media and publication. When participant countries are at position-D, they cannot misdemeanor/hide the medical data since every unique case has to be committed to a global ledger. By violating the protocol, they will have to answer to the UN, WHO, and other countries. When UNOs like WHO is at position-D, they cannot have a private dealership with an individual country after bringing this system into existence as every data is transparent and immutable to the whole world. Similarly, when position-D is occupied by news from global media and publication, irrespective of the country and their government, global media will get authenticate and precise information to keep the whole world on the same updated page.

global collaboration for its usability across a wide area of domains, providing limitless opportunities for fruitful areas for further exploration. It will also certainly extricate a blanket ban on information sharing to the world from tailored ideological straitjacket and thereby bring democracy and freedom. Last but not the least, epidemic or pandemic should have not actually occurred in functioning egalitarianism societies where the government is resilient to public opinion with transparency.

\section{CONCLUSION}

The digital ecosystem will wrestle with the world's greatest biological threats of infectious emergence or re-emergence through a coordinated and transparent approach with higher efficacy in collaborative sharing of information through IoT that

\section{REFERENCES}

Atzori, L., Iera, A., and Morabito, G. (2010). The internet of things: a survey. Comput. Netw. 54, 2787-2805. doi: 10.1016/j.comnet.2010.05.010

Beauchamp, J. (2013). Principles of Biomedical Ethics. New York, NY: Oxford University Press. doi: 10.1093/occmed/kqu158

Briscoe, G., and Wilde, P. (2006). "Digital ecosystems: evolving service-oriented architectures, in Conference on Bio Inspired Models of Network, Information and Computing Systems (Cavalese: IEEE Press). doi: 10.1145/1315843.13 15864

Car, J., Tan, W. S., and Huang, Z. (2017). eHealth in the future of medications management: personalisation, monitoring and adherence. BMC Med. 15:73. doi: 10.1186/s12916-017-0838-0

Chakravorty, A., and Rong, C. (2017). "Ushare: user controlled social media based on Blockchain," in Proceedings of the 11th International Conference on Ubiquitous Information Management and Communication (IMCOM). doi: $10.1145 / 3022227.3022325$

Fleming, N. (2018). How artificial intelligence is changing drug discovery. Nature 557:S55. doi: 10.1038/d41586-018-05267-x

Gold, M., and McLaughlin, C. (2016). Assessing HITECH implementation and lessons: 5 years later. Milbank Q. 94, 654-687. doi: 10.1111/1468-0009.12214

Heaven, D. (2019). Bitcoin for the biological literature. Nature 566, 141-142. doi: 10.1038/d41586-019-00447-9

Kaur, E., and Oza, A. (2020). Blockchain-based multi-organization taxonomy for smart cities. SN Appl. Sci. 2:440. doi: 10.1007/s42452-020-2187-4

Mirea, M., Wang, V., and Jung, J. (2019). The not so dark side of the darknet: a qualitative study. Secur. J. 32, 102-118. doi: 10.1057/s41284-018-0150-5

Pazaitis, A., Filippi, P., and de Kostakis, V. (2017). Blockchain and value systems in the sharing economy: the illustrative case of Backfeed. Technol. Forecast. Soc. Change 125, 105-115. doi: 10.1016/j.techfore.2017.05.025

Shilo, S., Rossman, H., and Segal, E. (2020). Axes of a revolution: challenges and promises of big data in healthcare. Nat. Med. 26, 29-38. doi: 10.1038/s41591-019-0727-5

Ting, D. S. W., Carin, L., Dzau, V., and Wong, T. Y. (2020). Digital technology and COVID-19. Nat Med. 26, 459-461. doi: 10.1038/s41591-020-0824-5

Topol, E. J. (2019). High-performance medicine: the convergence of human and artificial intelligence. Nat. Med. 25, 44-56. doi: 10.1038/s41591-018-0300-7 establishes a digital ecosystem with the data being utilized by bigdata analytics equipped with $\mathrm{AI}$ and blockchain technologies. The strategies of cohesion of a digital ecosystem of drug discovery to medication delivery and virtual clinics using AI-based algorithms can be amplified by bringing together the concerned organizations as nodes on chain to provide the accurate information. Thus, the digital ecosystem offers the automation, safety, and accountability by fast and transparent information sharing to the world about the provenance of pandemic and PHSM and to prevent any obfuscation or misdemeanor.

\section{AUTHOR CONTRIBUTIONS}

$\mathrm{AB}$ executed the design. $\mathrm{SB}$ conceived and contributed to the design. SK wrote and reviewed this article. All authors read and approved the final manuscript.

Udugama, B., Kadhiresan, P., Kozlowski, H. N., Malekjahani, A., Osborne, M., Li, V., et al. (2020). Diagnosing COVID-19: the disease and tools for detection. ACS Nano 14, 3822-3835. doi: 10.1021/acsnano.0c02624

Wikipedia. (2020). List of United Nations organizations. Available online at: https:// en.wikipedia.org/wiki/List_of_United_Nations_organizations (accessed May 17, 2020).

World Health Organization (2020a). Epidemic Intelligence from Open Sources (EIOS). Saving Lives through Early Detection. Available online at: https://www. who.int/eios (accessed May 14, 2020).

World Health Organization (2020b). Contact tracing in the context of COVID-19: interim guidance. Available online at: WHO/2019nCoV/Contact_Tracing/2020.1 (accessed on 14 May, 2020).

World Health Organization (2020c). "Novel Coronavirus (2019-nCoV) Situation Reports". Available online at: https://www.who.int/emergencies/diseases/ novel-coronavirus-2019/situation-reports (accessed 27 May, 2020).

World Health Organization (2020d). Critical Preparedness, Readiness and Response Actions for COVID-19 (Interim Guidance). Available online at: https://www. who.int/publications-detail/critical-preparedness-readiness-and-responseactions-for-covid-19 (accessed May 15, 2020).

World Health Organization (2020e). Operational Considerations for COVID-19 Surveillance using GISRS (Interim Guidance). Available online at: https:// www.who.int/publications-detail/operational-considerations-for-covid-19surveillance-using-gisrs-interim-guidance (accessed May 14, 2020).

World Medical Association (2020). Who We Are. Available online at: https://www. wma.net/who-we-are/ (accessed May 14, 2020).

Conflict of Interest: The authors declare that the research was conducted in the absence of any commercial or financial relationships that could be construed as a potential conflict of interest.

Copyright (c) 2021 Bharti, Krishnan and Bharti. This is an open-access article distributed under the terms of the Creative Commons Attribution License (CC BY). The use, distribution or reproduction in other forums is permitted, provided the original author(s) and the copyright owner(s) are credited and that the original publication in this journal is cited, in accordance with accepted academic practice. No use, distribution or reproduction is permitted which does not comply with these terms. 